\title{
Modeling the Isentropic Head Value of Centrifugal Gas Compressor using Genetic Programming
}

\author{
Ferozkhan Safiyullah ${ }^{1, a}$, Shaharin Anwar Sulaiman ${ }^{1}$, Nordin Zakaria ${ }^{2}$, Mohd Shahrizal Jasmani ${ }^{3}$, Syed
} Muhammad Afdhal Ghazali ${ }^{3}$

\author{
${ }^{1}$ Department of Mechanical Engineering, Universiti Teknologi PETRONAS, Tronoh, 31750, Malaysia. \\ 2 Department of Computer \& Information Sciences, Universiti Teknologi PETRONAS, Tronoh, 31750, Malaysia. \\ ${ }^{3}$ PETRONAS Carigali Sdn. Bhd., Kerteh, Terengganu, Malaysia.
}

\begin{abstract}
Gas compressor performance is vital in oil and gas industry because of the equipment criticality which requires continuous operations. Plant operators often face difficulties in predicting appropriate time for maintenance and would usually rely on time based predictive maintenance intervals as recommended by original equipment manufacturer (OEM). The objective of this work is to develop the computational model to find the isentropic head value using genetic programming. The isentropic head value is calculated from the OEM performance chart. Inlet mass flow rate and speed of the compressor are taken as the input value. The obtained results from the GP computational models show good agreement with experimental and target data with the average prediction error of $1.318 \%$. The genetic programming computational model will assist machinery engineers to quantify performance deterioration of gas compressor and the results from this study will be then utilized to estimate future maintenance requirements based on the historical data. In general, this genetic programming modelling provides a powerful solution for gas compressor operators to realize predictive maintenance approach in their operations.
\end{abstract}

Keywords: Genetic programming; gas compressor; computational model; predictive maintenance

\section{Introduction}

When a centrifugal gas compressor degrades in performance, the maintenance engineer will have to check the data from the gas compressor for possible and the root cause. The engineer will make a decision based on his experience and knowledge [1]. Besides that, the plant engineers do not know when the optimum time for them to do maintenance is. The engineer does not have a system to advise him on what might occur and the necessary steps to be taken. Normally, they will follow the Original Equipment Manufacturer's (OEM) recommendation, although they may probably enhance the compressor's life span thru a diagnostic system that constantly monitor and analyse the equipment's operation.

The objective of the present work is to develop a computational model, to derive the empirical relation for the isentropic head value of OEM performance map using the novel approach of genetic programming (GP). In OEM performance map the isentropic head is calculated

\footnotetext{
a Corresponding author: ferozkhan.safiyullah@nct.edu.om
}

by varying the inlet mass flow rate for different compressor rotational speed. The values of the isentropic head is extracted from the OEM performance map as output and the variable parameters inlet mass flow rate and the speed of the compressor are taken as the input variables.

Genetic programming is one of the most useful, general purpose problem solving techniques available to developers. It has been used to solve a wide range of problems, such as symbolic regression, data mining, optimization, and emergent behavior in biological communities. The scope of study in the present work is mainly on performance of the gas compressor due to fouling or blockage, whereas erosion and corrosion, mechanical damage, internal recirculation, and surge events will not be covered in this study. 


\section{Performance fundamentals}

Gas compressor performance are vital in oil and gas industry because of the equipment criticality which requires continuously operations. Currently, plant operators often face difficulties in predicting appropriate time for maintenance and would usually rely on timebased predictive maintenance intervals as recommended by Original Equipment Manufacturer [1]. Delayed decision on compressor maintenance intervention has caused prolonged downtime due to poor readiness of spare parts and resources. This work discussed on development of a computational model for predicting the isentropic head which is able to assist machinery engineers to quantify performance deterioration of gas compressor and predict optimum time for maintenance activities. Maintenance history data was collected from an oil producing offshore platform at the South China Sea are used as a case study for this project. The collected data is analysed regularly and maintenance advices are subsequently produced based on the input parameters. From the collected information, Genetic Programming Computational Model is developed which able to compute the fouling level of the compressor in terms of performance indicators deviations. The results are then being utilized to estimate future maintenance requirements based on historical data. In general, this Computational Model provides a powerful tool for gas compressor operators to realize predictive maintenance approach in their operations.

A gas compressor has a few parameters that are measurable. Examples are suction temperature and suction pressure. However, these parameters are difficult to be analyzed as the interpretation from the related data is inconsistent [2]. For instance, an increase in discharged pressure, unaffected discharged temperature and decrease in suction temperature. This does not provide any useful information when analyzed. Therefore, when analyzing parameters for gas compressors, performance indicator approach is more suitable. For the present work, only compressor isentropic head, will be discussed. These performance indicators are sufficient for data analysis. According to Sulaiman et al., "Isentropic head deviation monitoring is useful to identify decreasing trends of centrifugal compressor performance, when used in combination with other monitoring data plus computerized tools, the method can be a powerful analysis for compressor performance". Further Sulaiman et al., strongly recommends the usage of isentropic head deviation monitoring for maintenance interventions such as repair and overhaul. [1]. Undoubtedly, the head deviation monitoring plus powerful predictive modelling computerized tool such as genetic programming improves the compressor's performance.

Compressor head is the amount of energy applied for a unit amount of gas being compressed. It is often expressed in kilo joule per kilogram of gas $(\mathrm{kJ} / \mathrm{kg})$. Normally, isentropic head is related to pressure ratio and gas properties as:

$$
\mathrm{h}_{\text {isen }}=\mathrm{Z}_{\text {ave }} * \frac{R}{M W} * \frac{K}{K-1} * \mathrm{~T}_{\mathrm{s}} *\left[\left[\frac{P_{d}}{P_{S}}\right]^{\frac{K}{K-1}}-1\right]
$$

where $\boldsymbol{h}_{\text {isen }}$ is isentropic head in $\mathrm{kJ} / \mathrm{kg}, \boldsymbol{Z}$ ave is average compressibility factor, $\boldsymbol{R}$ is universal gas constant in
$\mathrm{J} / \mathrm{mol} . \mathrm{K}, \mathrm{MW}$ is molecular weight in $\mathrm{g} / \mathrm{mol}, \boldsymbol{k}$ is the ratio of specific heat, $\boldsymbol{T}_{\boldsymbol{s}}$ is the suction temperature in Kelvin, $\boldsymbol{P}_{\boldsymbol{d}}$ is discharge pressure in bar, and $\boldsymbol{P}_{\boldsymbol{s}}$ is suction pressure in bar. Fouling will cause the compressor head to decrease [1].The same equation can be applied to polytropic head, by replacing ratio of specific heats, $k$ with polytropic coefficient, $n$. Equation of state such as Redlich Kwong and Benedict- Webb-Rubin can be used to obtain the natural gas properties. However, industry uses Peng-Robinson and Soave-Redlich-Kwong equations of state and polytropic efficiency to determine natural gas properties and compressor performance respectively. They are slightly more accurate but more complex. The final selection of the equation of state will depend on the actual gas properties and process conditions and the most critical thing is to maintain consistency between all phases of testing and analysis [1].

\section{Genetic programming}

Genetic programming is better than a random search process. The symbolic regression function may be just too complicated to have been figured out by human trial and error functions. We might as well consider using Microsoft Excel 2003 with its data analysis Add-In. The problem is that such software and other specialized software merely fit in the co-efficient for a pre-determined polynomial or transcendental function. Symbolic regression is the process of discovering both the functional form of a target function and all of its necessary coefficient, or at least an approximation to these. This is distinct from other forms of regression such as polynomial regression in which you are merely trying to find the coefficients of a polynomial of a pre specified order [3]. Genetic programming is one instance of the class of techniques called evolutionary algorithms, which are based on insights from the study of natural selection and evolution [4]. Living things are extraordinarily complex; far more so than even the most advanced systems designed by humans. Evolutionary algorithms solve problems not by explicit design and analysis, but by a process akin to natural selection. An evolutionary algorithm solves a problem by first generating a large number of random problem solvers (programs). Each problem solver is executed and rated according to a fitness metric defined by the developer. In the same way that evolution in nature results from natural selection, an evolutionary algorithm selects the best problem solvers in each generation and breeds them [5,6]. Intuitively, if two computer programs are somewhat effective in solving a problem, then some of their parts probably have some merit. By recombining randomly chosen parts of somewhat effective programs, we may produce new computer programs that are even fitter in solving the problem. After the genetic operations are performed on the current population, the population of offspring (i.e., the new generation) replaces the old population (i.e., the old generation).Each individual in the population of computer programs in then measured for fitness and the process is repeated over many generations as shown in Figure 1. This process will produce populations of computer programs which, over many generations, tend to exhibit increasing average fitness in dealing with their environment. 
The best individual that appeared in any generation of a run (the best-so-far individual) is designated as the result produced by genetic programming. The hierarchical character of the computer programs that are produced is an important feature of genetic programming. The results of genetic programming are inherently hierarchical. In many cases, the results produced by genetic programming are default hierarchies, prioritized hierarchies of tasks, or hierarchies in which one behavior subsumes or suppresses another.

\section{Methodology}

There is a limitation in the availability of equipment as this study requires centrifugal compressors in operation. Hence, Universiti Teknologi PETRONAS (UTP) is working in collaboration with an oil company to provide the necessary equipment, data, and expertise in relation to this study. Data sets collected from the low pressure gas compressor were taken for the analysis. The compressor head deviation is our targeted output. Speed and inlet mass flow are taken as inputs. The data samples were randomized manually using Microsoft Excel software. The randomized data sets were fed into the software by initially splitting them into three sets viz., training, validation and applied testing [7]. DISCIPULUS ${ }^{\mathrm{TM}}$ selfconfigures itself to accept the last column always as the expected output. Trial runs to find out the best parameters that generated optimal solution in the minimum possible time. Initially the runs were performed with the default settings. One by one the parameters such as population size, crossover rate, DSS sub set size, and FPU registers used were varied to find optimum values [7]. The trials showed the following results. Population size of 800 was optimum rather than the default setting of 500. A higher crossover rate $(75 \%$ non- homologous and $25 \%$ homologous) was found to be optimum. A smaller DSS subset size (60) was more optimal than the default 100. The above factors favorably affected result generation.

\subsection{Regression}

Regression analysis is any statistical method where the mean of one or more random variables is predicted based on other measured random variables. Symbolic regression is the process of discovering both the functional form of a target function and all of its necessary coefficients, or at least an approximation to these.

\subsection{Fitness Measurement}

Fitness is nothing but how far the data value predicted by the genetic programming coincides with the experimental value

\subsection{Correlation Coefficient, r/R}

The quantity r, called the linear correlation coefficient, measures the strength and the direction of a linear relationship between two variables. The value of $r$ is such that $-1<r<+1$. The + and - signs are used for positive linear correlations and negative linear correlations, respectively. If $\mathrm{x}$ and $\mathrm{y}$ have a strong positive linear correlation, $r$ is close to +1 . An $r$ value of exactly +1 indicates a perfect positive fit. Positive values indicate a relationship between $\mathrm{x}$ and $\mathrm{y}$ such that as values for $\mathrm{x}$ increase, values for $\mathrm{y}$ also increase. . If $\mathrm{x}$ and $\mathrm{y}$ have a strong positive linear correlation, $r$ is close to -1 . An $r$ value of exactly -1 indicates a perfect negative fit. Negative values indicate a relationship between $\mathrm{x}$ and $\mathrm{y}$ such that as values for $\mathrm{x}$ increase, values for $\mathrm{y}$ also decrease. If there is no linear correlation or a weak linear correlation, $\mathrm{r}$ is close to 0 . The square of the correlation coefficient gives the coefficient of determination. The coefficient of determination, $\mathrm{r}^{2}$, is useful because it gives the proportion of the variance (fluctuation) of one variable that is predictable from the other variable. It is a measure that allows us to determine how certain one can be in making predictions from a certain model/graph. The coefficient of determination is the ratio of the explained variation to the total variation $[8,9]$. The coefficient of determination is such that $0<\mathrm{r}^{2}<1$, and denotes the strength of the linear association between $\mathrm{x}$ and $\mathrm{y}$. The coefficient of determination represents the percent of the data that is the closest to the line of best fit. If $r=0.922$, then $r^{2}=0.850$, which means that $85 \%$ of the total variation in y can be explained by the linear relationship between $\mathrm{x}$ and $\mathrm{y}$. the other $15 \%$ of the variation in $\mathrm{y}$ remains unexplained.

\section{Results and Discussions}

In genetic programming modeling, it is necessary to select suitable terminal from set $F$ and available terminal genes from set $f(0)[10,11]$. From these, the evolutionary process will try to build as fit an organism (i.e. mathematical model) as possible for characteristics prediction. The organisms consist of both terminal and function genes, having the nature of computer programs which differ in form and size $[12,13]$. Three independent data sets were obtained on the basis of measurement: training, validation, applied data sets. Speed and inlet mass flow rate were used as independent input variables and the isentropic head as dependent output variable. On the basis of training data set, different models for isentropic head were developed by the genetic programming $[14,15$, and 16]. Using genetic programming simulation, the best mathematical model for Compressor isentropic head is given by

Isentropic Head $=\sqrt{\left[\sin \left(4 \sin D \times V_{1}\right)+V_{0}-E\right]}$

Where,

$$
\begin{aligned}
& \mathbf{E}=-23.824 \mathrm{~A}+3 \sqrt{2 C}+\mathrm{D}-\left(4 \sin D \times \mathbf{V}_{\mathbf{1}}\right) ; \\
& \mathbf{D}=\sqrt{(2 \sqrt{2 C}-11.912 \mathrm{~A})} ; \\
& \mathbf{C}=9.96\left(\mathrm{~B}^{2}+11.912 \mathrm{~A}\right)+\mathbf{V}_{\mathbf{0}}-11.912 \mathrm{~A} ; \\
& \mathbf{B}=\frac{10.912 A+\mathbf{V}_{\mathbf{0}}}{0.4943 \mathbf{V}_{\mathbf{1}}} ; \mathbf{A}=\cos \left[\frac{1.5394 \mathbf{V}_{\mathbf{0}}}{\mathbf{V}_{\mathbf{1}}}\right]
\end{aligned}
$$

$\mathbf{V}_{\mathbf{0}}=$ Speed of the Compressor (in RPM); $\mathbf{V}_{\mathbf{1}}=$ Inlet mass flow rate (in $\mathrm{m}^{3} / \mathrm{hr}$ ). In order to simplify Eq. (1), there is some necessity to introduce some intermediate variables likes A, B, C, D and E. The theoretical isentropic head 
values can be calculated by back substitution. Initially the values of intermediate variables should be calculated and then finally substitute in Eq. (2) to get the isentropic head values. The calculated value from the OEM performance chart is compared with the predicted values generated from the above empirical relation for the isentropic head. Fig. 1 shows the graph, how far the values of isentropic head from the OEM performance chart and predicted values coincides with each other for different speeds. In Fig. 1 ' $N$ ' denotes the speed of the compressor. Based on the deviation of the predicted isentropic head value from the OEM performance chart value, an error graph is drawn below. The maximum predicted error value is $3.9 \%$ and the minimum is $0 \%$. The average predictive error value is $1.318 \%$ as shown in Fig. 2. This shows predicted value from the computational model developed from the genetic programming concept almost matches with the isentropic head value calculated from the thermodynamic equation and the correlation is shown in Fig. 3.
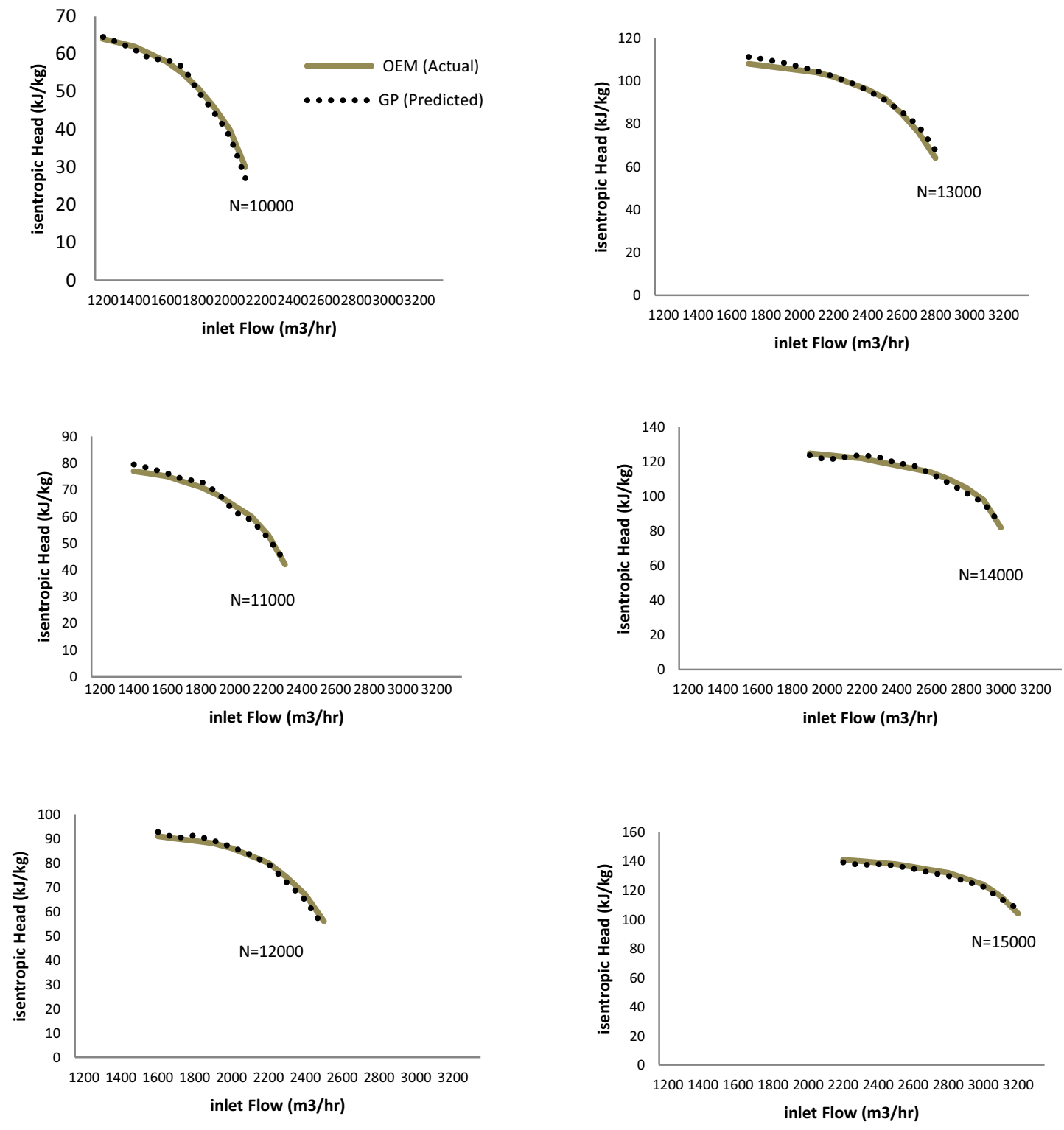

Fig 1: Variation of actual and predicted isentropic head for different speeds 


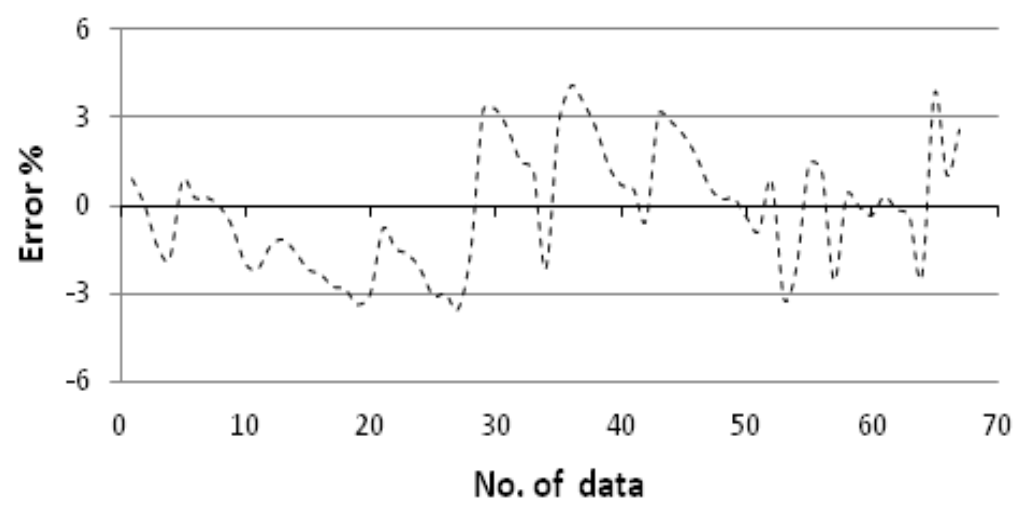

Fig 2: Error Graph

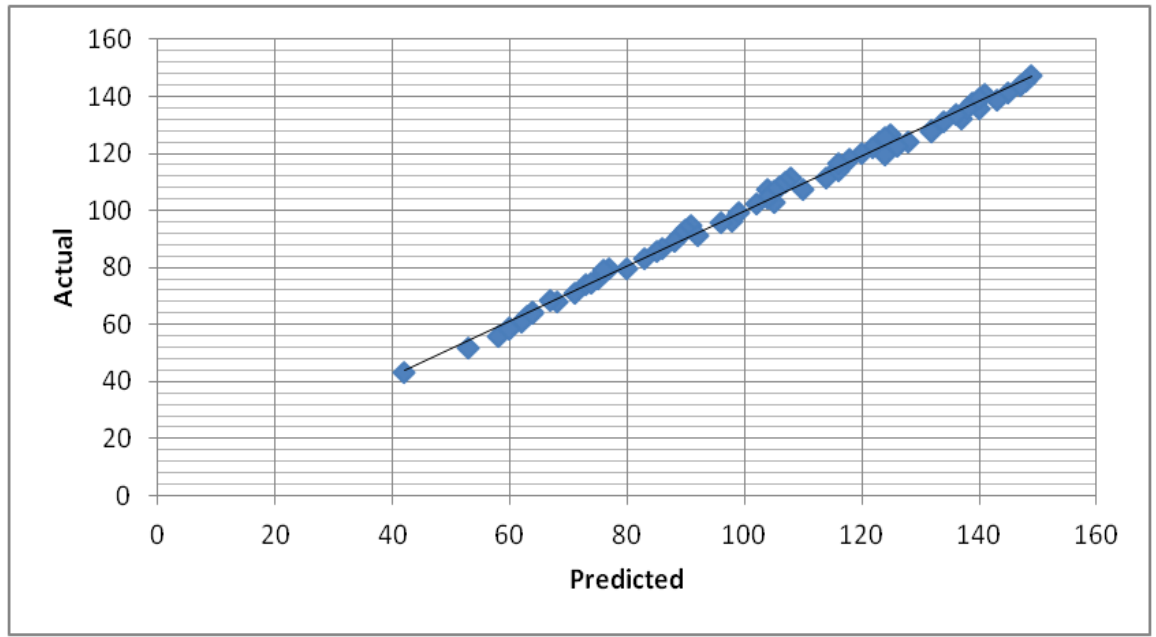

Fig 3: Relationship between actual and predicted isentropic head

\section{Conclusions}

Using the above computational model we will predict the compressor head for various speeds and inlet mass flow by simple substitution. Genetic programming (genetic programming) has proved to be a highly versatile and useful tool for identifying relationships in data for which a more precise theoretical construct is unavailable $[17,18]$. The experimental data in this research were in fact the environment to which the population of models had to be adapted as much as possible. The models presented are a result of the self organization and stochastic processes taking place during simulated evolution. The accuracies of solutions obtained by genetic programming depend on applied evolutionary parameters and also on the number of measurements and the accuracy of measurement. In general, more measurements supply more information to evolution which improves the structures of models. In the proposed concept the mathematical models for verifying the experimental results of mechanical characteristics are subject to adaptation. Its reliability is about $99.26 \%$. In the testing phase, the genetically produced model gives the same result as actually found out during the experiment, thereby with the reliability of cent percent. It is inferred from our research findings that the genetic programming approach could be well used for the prediction of various gas compressor readings without conducting the experiments. This helps to establish efficient planning and optimizing of process for the quality production depending upon the functional requirements.

\section{Acknowledgements}

The authors would like to convey our gratitude to PETRONAS Carigali Sdn. Bhd for the permission to utilize the proprietary data, information used in this publication. 


\section{References}

[1] Shaharin Anwar Sulaiman, Khairil Ikhwan, Mohd Shahrizal Jasmani., development of gas compressor diagnostic program using knowledge basedmanagement concept Proceedings of the ASME 2013.IMECE.(2013)

[2] Meher-Homji, C.B., Boyce, M.P., Lakshminarasimha,A.N., Whitten, J. \& MeherHomji, F.J., 'ConditionMonitoring and Diagnostics Approaches for Advance GasTurbines', ASME COGEN-TURBO (IGTI), Vol.8, pp.347-354.( 1993).

[3] Ferozkhan Safiyullah, Mohammed Irfan, Aslam Amirahmad., IOSRJMCE. ISSN: 2278-1684 Volume 2, Issue 1, PP 46-50 (2012).

[4] Koza, J.R., (1992) Genetic Programming: On the Programming of Computers by Natural Selection.MIT Press, Cambridge, MA.

[5] J. R. Koza, Genetic programming II, The MIT Press, Massachusetts, (1994).

[6] Koza, Bennett, Andre, \& Keane, GENETIC PROGRAMMING III - Darwinian Invention and Problem Solving, Morgan Kaufmann Publishers, Inc. pp. 1154. (1999).

[7] Francone, F., Discipulus Owner's Manual and Discipulus Tutorials, Register Machine Learning Technologies, Inc. (1998-2000).

[8] M. Kovacic, J. Balic and M. Brezocnik, Evolutionary approach for cutting forces prediction , Journal of materials processing technology, 155/156, (2004).

[9] H. Kurtaran, B. Ozcelik and T. Erzurumlu, Warpage optimization of a bus ceiling lamp base using neural network model and genetic algorithm, Journal of materials processing technology, 169(2), (2005).
[10] Sette S., Boullart L. Genetic programming: principles and applications, Engineering Applications of Artificial Intelligence 14 (2001).

[11] Pierreval H., Caux C., Paris J.L., Viguier F. Evolutionary approaches to the design and organization of manufacturing system, Computers \& Industrial Engineering 44 (2003).

[12] Gusel L., Brezocnik M. Modeling of impact toughness of cold formed material by genetic programming, Comp. Mat. Sc. 37 (2006).

[13] Chang Y.S., Kwang S.P., Kim B.Y. Nonlinear model for ECG R-R interval variation using genetic programming approach, Future Generation Computer Systems 21, pp. 1117-1123.(2012).

[14]Brezocnik M., Gusel L. Predicting stress distribution in cold formed material with genetic programming, International Journal of Advanced Manufacturing Technology, Vol. 23, pp.467-474. (2004).

[15]M. Brezocnik, M. Kovacic and M. Ficko, Prediction of surface roughness with genetic programming, Journal of materials processing technology, 157/158, 28-36. (2004).

[16] M. Brezocnik and M. Kovacic, Integrated genetic programming and genetic algorithm approach to predict surface roughness, Materials and manufacturing processes, 18(4) (2003).

[17] Mohammed Yunus, J. Fazlur Rahman and S.Ferozkhan, Genetic programming approach for the prediction of thermal characteristics of ceramic coatings, IJIERD, Volume 2 Issue 1, May - October (2011).

[18] Mohammed Yunus, J. Fazlur Rahman and S.Ferozkhan, Evaluation of machinability characteristics of industrial ceramic coatings using genetic programming based approach, IJMET, Volume 2, Issue 2, August- December (2011). 